\title{
Influence of Flexibility on the Separation of Chiral and Structural Isomers in the STW-Type Zeolite
}

\author{
Rocio Bueno-Perez ${ }^{\mathrm{a}}$, Salvador R. G. Balestra ${ }^{\mathrm{a}}$, Miguel A. Camblor ${ }^{\mathrm{b}}$, Jung Gi Min ${ }^{\mathrm{c}}$, Suk Bong \\ Hong $^{\mathrm{c}}$, Patrick J. Merkling ${ }^{\mathrm{a}^{*}}$, Sofia Calero ${ }^{\mathrm{a}}$ * \\ ${ }^{a}$ Department of Physical, Chemical and Natural Systems.Universidad Pablo de Olavide, Crtra. De Utrera km. 1, 41013 \\ Seville (Spain) \\ ${ }^{\mathrm{b}}$ Instituto de Ciencia de Materiales de Madrid (ICMM), Consejo Superior de Investigaciones Científicas (CSIC), Sor \\ Juana Inés de la Cruz 3, 28039 Madrid (Spain) \\ ${ }^{\mathrm{c} C e n t e r ~ f o r ~ O r d e r e d ~ N a n o p o r o u s ~ M a t e r i a l s ~ S y n t h e s i s, ~ D i v i s i o n ~ o f ~ E n v i r o n m e n t a l ~ S c i e n c e ~ a n d ~ E n g i n e e r i n g, ~}$ \\ POSTECH, 37673, Pohang (Korea) \\ KEYWORDS. Flexibility, Enantioselectivity, Preferential adsorption, Molecular simulation
}

\begin{abstract}
Molecular simulation is a useful tool to predict the selective capacity of nanoporous materials by calculating adsorption isotherms. Generally, adsorbents are modeled as rigid frameworks, as opposed to allowing for the vibrations of the lattice, and the effect on adsorption is assumed to be negligible. In this work we test this approach in an especially challenging system by computing the adsorption of the chiral molecules 2-pentanol, 2-methylbutanol and 3-methyl-2butanol in the all-silica and silicogermanate chiral zeolites STW, and their lattice vibration upon adsorption. The analysis of single and multicomponent adsorption isotherms shows the suitability of the STW-type zeolites as molecular sieves for chiral and structural separation processes, which pose a challenging task in the chemical and pharmaceutical industries. We also provide new experimental adsorption data that validate the force field employed. Our results reveal that the lattice vibration of the all-silica framework is limited and maintains the confinement of the chiral environment, which drives the selection of R-2-pentanol over the S enantiomer. The lattice vibrations of silicogermanate STW on the other hand display host-guest coupling modulated by uptake and sorbate type that disrupt the chiral recognition sites. These structural distortions, however, maintain the preferential adsorption of this framework towards 2-pentanol over the other structural isomers. This study points out that the effects of intrinsic flexibility on the selective capacity of nanoporous materials may range from small to high impact, some of them could not have been foreseen even after the examination of the structural dynamics of an empty framework.
\end{abstract}

Biological systems, from the very lowest to the highest life forms, are homochiral entities that fulfill a broad variety of functions through a complex network of three-dimensional structure-activity interactions performed by enantiopure compounds. ${ }^{1}$ As a consequence, any technology or technical advance derived from or focused on biosystems should take these complex interactions into account. Indeed, as nature is increasingly dealing with synthetic chemicals, the awareness of chiral toxicity is increasing as well. The most representative cases are featured in the pharmaceutical industry ${ }^{2}$ where only one enantiomer of the racemic drug is therapeutically effective while the other is inactive or might even have undesired effects, which might be just different or, in the worst case, adverse. Likewise, in the food industry the chirality of a flavor enhancer results in a compound being tasty or tasteless, as happens with monosodium glutamate; and in the perfume industry chirality is also key since depending on the chiral form different organoleptic properties are achieved. ${ }^{3}$ Prominent examples of this are the distinct fragrances of limonene or menthol. Finally, following the same trend, the agrochemical industry is improving the safety of herbicides, as many of them are chiral and still used as racemates, which can have different effects on target weeds and non-target organisms. ${ }^{4}$ Thus, chiral toxicology has become an area of study which is still relatively unexplored and is a relevant issue in several chemical manufacturing industries. However, the synthesis of enantiopure compounds is difficult and costly, and many approaches have been proposed from the areas of enzymatic and chemical synthesis. The enzymatic synthesis takes advantage of the specificity of enzymes and metabolic routes, yet scaling it up to increase its performance involves long-term research and, moreover, is not transferable. ${ }^{5,6}$ Chemical synthesis is less specific but achieves higher yields and, combined with the development of chromatographic separation techniques, enantiomeric resolution is possible. Specifically, high-performance liquid chromatography (HPLC) is currently the most widely used technique for analytical purposes and preparative separations of chiral compounds. ${ }^{7,8}$ Recently, special attention has been drawn to the role of stereoregular polymers as chiral stationary phases (CSPs) among which crystalline nanoporous materials are found ${ }^{8}$. Several studies report the chiral recognition skills of metal-organic frameworks (MOFs) ${ }^{8,10-12}$ and zeo- 
lites $^{13-17}$. These studies analyze in depth the molecular mechanisms for chiral recognition and rationalize the features of these materials that relate to several enantioselective behaviors such as, selective adsorption ${ }^{13-15,18-20}$ or asymmetric cataly$\operatorname{sis}^{16,21-23}$. As a consequence, many efforts have been put on the rationalized design of new chiral materials and the methods for their synthesis ${ }^{24-31}$.

Up to now, a small number of zeolite frameworks have been crystallized in chiral space groups. Among them, STW is a recently discovered zeolite that was first synthesized as silicogermanate zeolite called SU- $32^{32}$. This material was difficult to prepare in a pure form and suffered from the thermal and hydrothermal stability problems typical of germanosilicates. Afterwards, a different synthetic strategy afforded a pure-silica zeolite with the same STW framework type ${ }^{33,34}$, known as HPM-1 zeolite, which has the high thermal and hydrothermal stability of silica materials. The pore topology of the STW zeotype can be described as a helicoidal channel running along the $z$-axis, which consists of six rectangular cages oriented in the three dimensions of space and perpendicular to the direction of the channel (Figure 1). Each cage is rotated $60^{\circ}$ to the right of the previous one in space group $\mathrm{P}_{1} 22$, all of them connected through $10-$ membered rings ${ }^{21}$. There is, of course, an enantiomeric crystal in space group $\mathrm{P}_{5} 22$, in which the cages are rotated to the left and, in principle, the actual crystalline powder is racemic conglomerate. The helicoidal channel defined is intersected by 8 -membered rings which shape the cages. Given the chiral topology of this zeotype, and the fact that individual crystals are enantiopure ${ }^{35}$, it is a useful study model on enantioselectivity connected with potential applications $^{13}$. This idea is reinforced by the very recent report of the synthesis of enantioenriched germanosilicates with the STW structure using enantiopure organic structure-directing agents and the evidence of significant chiral selectivity over the resulting enriched material in both catalysis and adsorption processes $^{36}$. Likewise, the arrangement of cages and channels defines a channel system that seems also suitable for the structural selectivity of compounds with different structural features, such as branched and linear alkanes and alcohols ${ }^{37-39}$.

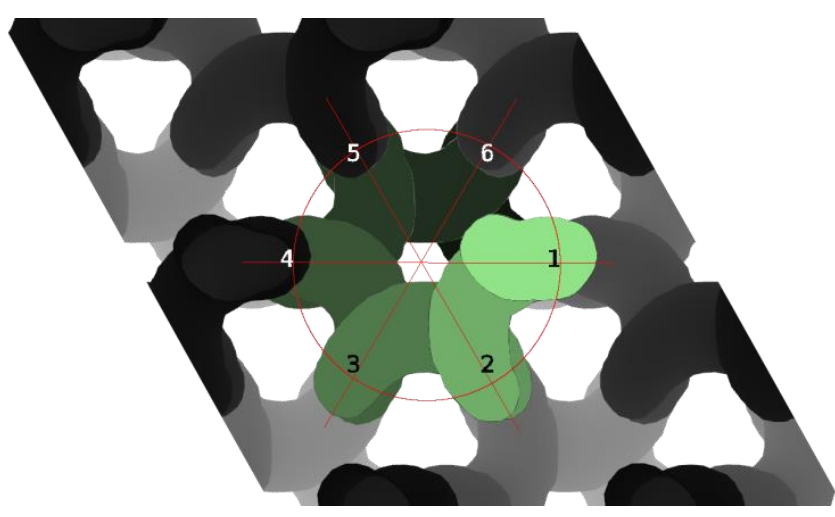

Figure 1. XY-view of the chiral pore topology of one enantiomer of zeotype STW, which consists of six rectangular cages oriented in the three dimensions of space with a rotation of $60^{\circ}$ along the $z$-axis.

One of the most widespread ideas is that chiral recognition is led by the confinement of the molecule inside the pores or channels of the materials ${ }^{14,15,40}$. Along with the experimental results related to enantioselective separation which reveals the actual potential of chiral nanoporous materials as molecular sieves and CSPs ${ }^{10,11}$ theoretical studies based on molecular simulations unravel the underlying molecular mechanisms. In addition to the close match of the molecule to the size of the channel, other factors related to guest-guest and host-guest interactions must be considered. Some materials, such as homochiral MOFs ${ }^{18}$, might have more than one chiral recognition site based on host-guest interactions. These sites could be selective to the opposite enantiomer, or the same which would increase chiral selectivity ${ }^{20,41}$. Also the channel surface chemistry might change the affinity with which the molecules are adsorbed within the pore, influencing the confinement and selectivity consequently ${ }^{42}$. Regarding guest-guest interactions, dispersive forces, dipole-dipole interactions, and hydrogen bonding may be especially ineffective for the same or the opposite enantiomer inside the channel and determine the packing of enantiomers and, therefore, the selectivity ${ }^{11}$. As an example of the relevance of the overall contribution of the aforementioned factors, additional mechanisms exist that allow non-chiral materials to be enantioselective in non-racemic mixtures. In the "random cell" mechanism, the unordered packing of one enantiomer leaves asymmetric spaces that can be occupied by the opposite enantiomer ${ }^{43}$. In the case of the "chiral cell" mechanism, these asymmetric spaces are generated by extra-framework cations ${ }^{44}$. To conclude, the key to the chiral selectivity is the confinement, i.e., the adequate match of size and shape between the molecule and the channel, though this factor can be considered a consequence of or modulated by the contribution of host-guest and guest-guest interactions related to the features of a given material.

In the context of the relationship between confinement and chiral selectivity, the flexibility of porous crystalline materials can have a profound effect. Some of them undergo flexible phenomena $^{45}$ such as breathing ${ }^{46}$, swelling ${ }^{47,48}$, linker reorientation $^{49,50}$, negative/positive thermal expansion ${ }^{51}$ or subnetwork displacement ${ }^{52}$, which are generally triggered by external stimuli, such as temperature, pressure or guests adsorption ${ }^{12}$. But for most frameworks, this intrinsic flexibility ${ }^{53}$ involves just lattice vibrations and does not affect adsorption properties or the crystalline regularity strongly. These frameworks are commonly considered as rigid and zeolites are typically ascribed to this group. However, the intrinsic flexibility of a zeolite might also induce changes in the pore opening able to disrupt the structural selectivity of this material ${ }^{54}$. Several studies were performed to elucidate its real implication through diffusion kinetics of several compounds of different sizes and chemical nature ${ }^{55-59}$. The results indicate an increase in the diffusion values as a consequence of less energetic cageto-cage crossings allowed by the vibration of the window ring and point to the relevance of this effect when the adsorbate fits tightly into the pore ${ }^{54,59}$. Other studies ${ }^{60}$ claim that lattice vibrations might affect pore opening and diffusion kinetics in different ways with very different underlying mechanisms. Another aspect to bear in mind is that changes in the chemical composition can also alter the flexibility pattern of a framework since the presence of germanium in zeolites confers static and dynamic flexibility ${ }^{58}$ to an otherwise rigid framework. Considering this, the interplay between flexibility and confinement might alter dramatically the chiral recognition capacity of a framework. Recently, several studies have focused on the development of efficient methods to approach computationally the effects of flexibility on adsorption properties, which otherwise lead to very time-consuming simulation ${ }^{56}$. 
Unfortunately, it is not clear yet how accurately these methods capture the flexibility effects without having to simulate a fully flexible framework, and reporting the behavior of the framework upon adsorption is likely to be relevant in the case of chiral selectivity.

The current work presents simulation results of an extensive research on chiral and structural separation in chiral flexible zeolites. Both the all-silica and silicogermanate frameworks of the chiral zeotype STW are used as adsorbents. The chiral probes chosen are the chiral isomers belonging to the group of amyl alcohols: 2-pentanol (2P), 2-methylbutanol (2MB) and 3methyl-2-butanol (3M2B). The $R$ enantiomer of $2 \mathrm{P}$ is widely used as an intermediate in the synthesis of drugs against Alzheimer's disease ${ }^{61} .2 \mathrm{MB}$ and $3 \mathrm{M} 2 \mathrm{~B}$ are structural isomers of $2 \mathrm{P}$ with methyl groups in different relative positions, which allows to study the molecular arrangement induced by guestguest interactions on the chiral separation.

\section{Simulation details}

The structures for HPM-1 and SU-32, all silica and silicogermanate frameworks of STW, respectively, were built through a meticulous process of energy optimization using the Vienna Ab-Initio Simulation Package (VASP) to get the most stable structure of each configuration ${ }^{62,63}$. The two sets of experimental atomic positions reported for the all-silica ${ }^{34}$ and the silicogermanate frameworks ${ }^{32}$ were geometrically optimized while keeping the cell parameters fixed. To this aim, previous1y, 20 assumed silicogermanate structures were constructed from the experimental set of SU-32 positions based on distributions of silicon and germanium atoms in agreement with the reported populations in each crystallographic site ${ }^{32}$. The energy of these structures were calculated with VASP by singlepoint calculation using electronic correlations and the functional PBEsol ${ }^{64}$, from which the one with the lowest energy is retained. Finally, the all-silica and the selected silicogermanate structures were relaxed with VASP, optimizing both atomic positions and cell parameters. The resulting structures are used in this study.

To study the chiral recognition capacity of the adsorbent, the asymmetric carbon that confers chirality to the guest molecules must be properly defined. Therefore, an all-atom flexible force field is used to model each pair of enantiomers for each of the alcohols (Figure $\mathrm{S} 1$ in the Supporting Information): $R / S$ 2-pentanol (2P), $R / S$-2-methylbutanol (2MB) and $R / S-3$ methyl-2-butanol (3M2B). Thus, the alcohol adsorbates are flexible and based on Lennard-Jones (L-J) parameters, charges and geometries defined by OPLS-AA force field ${ }^{65}$ (Table S1 and $\mathrm{S} 2$ in the Supporting Information). To define the hostguest interactions, Lorentz-Berthelot mixing rules were applied to the L-J parameters of guests and host. Charges and L-J parameters of silicon and oxygen atoms of the host were defined through the TraPPE force field specific for zeolites ${ }^{66}$. L$\mathbf{J}$ parameters and charge of germanium atoms are assumed equal to silicon atoms since the valences of silicon and germanium are the same, and their electronegativity and polarizability are rather similar ${ }^{14,67}$. Both HPM-1 and SU-32 structures are modelled either as rigid or as flexible. When rigid the crystallographic positions of each atom are fixed in space. When the structure is considered flexible, Hill-Sauer ${ }^{68}$ force field is used to model the host-host interactions, bond stretching, angle bending and torsions in the framework, since it has been efficiently used for similar computational works ${ }^{49,56}$. For this work, geometric parameters of Hill-Sauer were adjusted to consider the Ge-O bond distance $(1.77 \AA)$, which is longer than the Si-O bond (1.61 $\AA$ ). Charges, L-J parameters and models for bond-stretching, angle-bending and torsions in hosts and guests are detailed properly in Tables S3-S4 of the Supporting Information.

Adsorption data of each enantiomer of $2 \mathrm{P}, 2 \mathrm{MB}$ and $3 \mathrm{M} 2 \mathrm{~B}$ for both single-components and mixtures were computed using Grand Canonical Monte Carlo simulations (GCMC) at 298 $\mathrm{K}$ with the RASPA software ${ }^{69}$. A $2 \times 2 \times 1$ super cell was chosen for the simulation box for both all-silica and silicogermanate STW structures (STW-Si and STW-SiGe), given that the L-J cutoff radius was set to $12 \AA$, all the dimensions of the simulation box are larger than twice the cutoff radius. Periodic boundary conditions were applied ${ }^{70}$ and long-range electrostatic interactions calculated through Ewald summations. Simulations were arranged in cycles of trial moves: insertions and deletions based on the Continuous Fractional Component move ${ }^{71}$, total and partial reinsertions, random translations and rotations of the molecules. Each point of the isotherms computed for single components were obtained from the average of four independent simulations after $10^{5}$ cycles of initialization and $10^{6}$ cycles of production. The adsorption values for binary mixtures of structural isomers were computed in the same way. For enantiomeric mixtures, S-curves at $10^{6} \mathrm{~Pa}$ were calculated. An S-curve displays a plot in which each point represents the molar fraction of adsorbed molecules for a given enantiomer against the molar fraction fixed in the bulk for the same enantiomer. Each point on this type of graph is obtained as the average of eight independent simulations after $10^{4}$ cycles of initialization and 500000 cycles of production. For these mixtures, simulations were speeded up by introducing an additional energy-biased identity change move with the same probability as other intended moves.

Every simulation was performed for both rigid and flexible frameworks. In the latter, insertion moves are handled with the Configurational-Bias insertion move $\mathrm{e}^{72}$. To study the structural flexibility upon adsorption and to ensure a proper host-guest interaction we use a MC-move which inserts a short Molecular Dynamics (MD) simulation in the microcanonical (NVE) ensemble in a hybrid Grand Canonical Monte Carlo algorithm (here called hybrid MCMD). This allows for the flexibility of the framework without changing its volume. Because of the ill-defined nature of simulating both the molecular adsorption and framework thermal coupling with the inserted/deleted molecule, this kind of algorithm is crucial to capture the distortions upon the guest-adsorption process ${ }^{73-75}$. In all of the simulations performed incorporating MD moves, pure compound isotherms, adsorption isotherms of binary mixtures and S-curves, each point was obtained after 15000 cycles of initialization, 300000 cycles of production and an overall time of framework dynamics of $2 \mathrm{~ns}$. From these simulations, framework snapshots were taken to study the behavior of intermediate frameworks.

Diffusion data were obtained through MD simulations in the canonical ensemble, at $298 \mathrm{~K}$ and infinite dilution, with one single molecule moving inside the simulation supercell. The time step was set to $0.5 \mathrm{fs}$ and the mean squared displacement (MSD) of the molecule was recorded for $250 \mathrm{~ns}$ on average. These simulations were performed for each enantiomer of the three alcohols considering a rigid framework. The MSD were fitted to a linear regression to obtain diffusion coefficients 

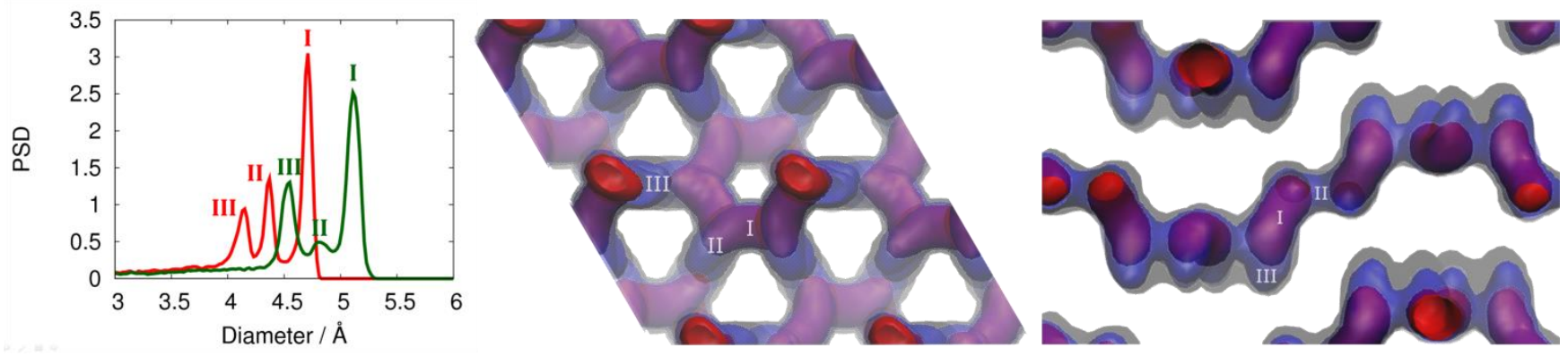

Figure 2. Pore size distribution (left) of STW-Si (red) and STW-SiGe (green). XY (center) and ZY (right) views of STW geometry showing the internal pore space featuring wide sections (I) in red, diffusion limiting necks (II) and pockets (III) in blue. These views were generated with Pore Blazer by moving probe particles of increasing diameter sizes in STW-Si: $3.5 \AA$ (grey silhouette), $4 \AA ̊$ (blue area) and $4.5 \AA ̊$ (red area).

for each enantiomer in each structure ${ }^{76}$. Likewise, MD simulations were also carried out in the canonical ensemble, at $298 \mathrm{~K}$ with a time step of $0.5 \mathrm{fs}$, to study the flexibility pattern of empty STW-Si and STW-SiGe up to $5 \mathrm{~ns}$.

To shed light on the effects exerted by the vibration of the frameworks we computed the average minimum aperture distribution of 8 and 10-membered rings, i. e., the time average of the minimum O-O separation across the aperture. This calculation was performed for each snapshot generated during the hybrid MCMD simulations that provided the singlecomponent adsorption isotherms and the adsorbed fractional content of $R$ enantiomer from racemic mixtures. The resulting values were averaged and shown by component. The reference used in the comparison is the average minimum aperture obtained from the snapshots from MD simulations carried out with the empty frameworks. Furthermore, to gain additional insight we calculated the pore size distributions with RASPA and obtained a detailed view of the accessible space inside the structures using Pore Blazer software ${ }^{77}$ to move probes of different diameter sizes along the channels. This software considers the van der Waals radii of framework atoms and checks the connectivity of channels by using probes of a given diameter. Simulations in the canonical ensemble were also run for a single molecule moving randomly (molecular translation, rotation and reinsertion) to compute average occupational density profiles, and for a total number of adsorbed molecules of 4,8 , $12,16,20,24$, and 28 versus a racemic bulk to study the loading-dependent enantiomeric composition in the framework. In the latter simulations, random moves and identity change operations were permitted. Simulations were performed five times independently using the rigid STW-Si and STW-SiGe and their intermediate frameworks taken from the hybrid MCMD simulations run for the adsorption of chiral mixtures. The simulations carried out in this study were mostly for the right handed STW structures (space group P6 22 ). However, to test the enantioselectivity in the inverted structures complementary simulations were also conducted to obtain the Scurve for the enantiomeric mixtures of $2 \mathrm{P}$ and $3 \mathrm{M} 2 \mathrm{~B}$ in the left handed all silica and silicogermanate rigid structures $\left(\mathrm{P}_{5} 22\right)$.

\section{Experimental Methods}

Pure silica HPM-1 (STW) was synthesized according to previously reported procedures using 2-ethyl-1,3,4trimethylimidazolium and fluoride as structure-directing agents $^{33,34}$. Adsorption data for 1-pentanol, 2-pentanol, and 3pentanol on HPM-1 were obtained volumetrically at $313 \mathrm{~K}$ and pressure ranging from 0 to 10 Torr using 3Flex Micromeritics analyzer. The system guaranteed high accuracy with a resolution of 10-5 Torr. The temperature within the chamber was maintained at a constant value with a precision of $\pm 0.3 \mathrm{~K}$. The purity of all organic adsorbates studied here were higher than $99.8 \%$, purchased from Sigma-Aldrich. Each of the three pentanol isomer vapors was generated by heating its sample were placed into the cylindrical quartz bucket (flat bottom, $9 \times 12$ $\mathrm{mm}$ ) and evacuated to a residual pressure of 10-3 Torr at 523 $\mathrm{K}$ for $6 \mathrm{~h}$. A point isotherm was recorded at equilibrium when no further uptake change was observed. The data were not corrected for non-ideality.

\section{Results and Discussion}

In this work we consider two materials with different chemical compositions but the same STW zeolite topology and the same space group of symmetry $\left(\mathrm{P}_{6} 122\right.$ or $\mathrm{P}_{5} 122$, \#178 and $\# 179$, respectively, depending on the chiral configuration) so that the characteristic chiral channel of this topology is present in both frameworks. As explained in the simulation details section the silicogermanate framework has been generated based on the relative population of $\mathrm{Si} / \mathrm{Ge}$ atoms in each one of the five crystallographic sites ${ }^{32}$ with an overall ratio $\mathrm{Si} / \mathrm{Ge} \sim 1$. The cell parameters of the silicogermanate framework (STW$\mathrm{SiGe}$ ) are $4 \%$ larger than those of the all-silica structure (STW-Si), as depicted in Table $5 \mathrm{~S}$ of the Supporting Information. The accessible pore volume is lower for STW-Si $\left(1297.49 \AA^{3}\right)$ than for STW-SiGe, (1583.99 $\left.\AA^{3}\right)$ and account for approximately $34 \%$ and $37 \%$ of the total volume of each unit cell, respectively. Even though the two frameworks share the same topology, their correspondence is not merely obtained by scaling, as can be seen in Figure 2 (left) from the pore size distribution (PSD). Three discernible peaks are found and the ones for STW-SiGe 

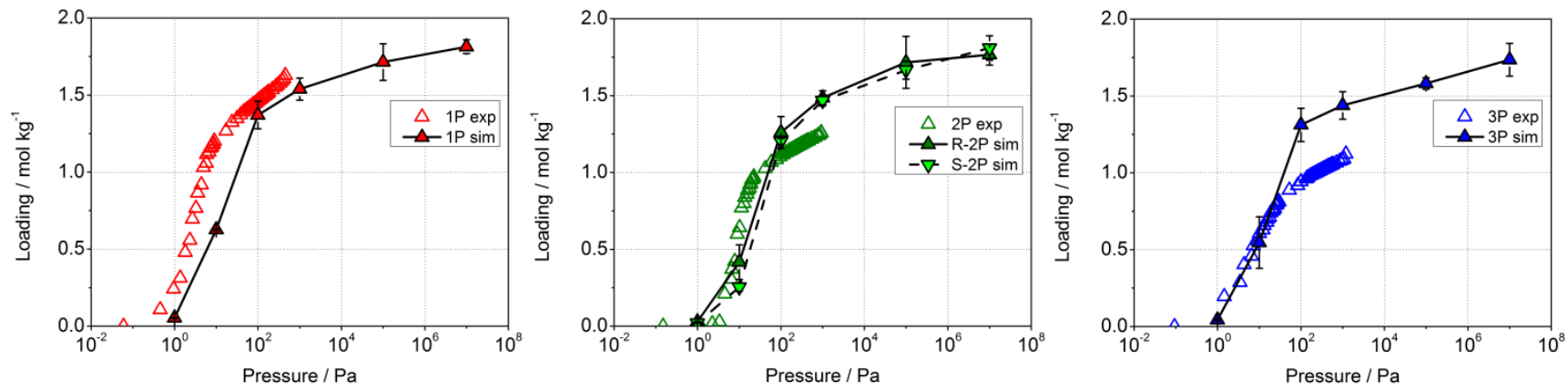

Figure 3. Adsorption isotherms of ${ }_{1} \mathrm{P}, R / S-2 \mathrm{P}$ and ${ }_{3} \mathrm{P}$ in $\mathrm{STW}-\mathrm{Si}$ at $313 \mathrm{~K}$ : comparison of experimental data (open symbols) and data obtained computationally (full symbols).

are displaced circa $0.4 \AA$ to the right. STW-Si shows three peaks at increasing diameters: $4.15,4.37$ and $4.71 \AA$. STWSiGe shows also three peaks at $4.5,4.81$ and $5.11 \AA$, although the intensity of the medium-sized peak is low compared to the others. Since the topology of STW consists of onedimensional chiral channels, the PSD describes sections of different sizes inside the pore. To study the accessibility through these pore sections the void space distribution inside the frameworks has been computed using Pore Blazer and analyzed. Thus, probe particles with increasing diameter sizes, from 2 to $7 \AA$, were used to explore the pore. The pore space of STW-Si is visualized in Figure 2 for probe particles of 3.5 (grey), 4 (blue) and $4.5 \AA$ (red). These diameters were selected because they allow for a clear view of each pore section, which are analogous in both structures. The largest diameter for which a probe particle can run along the channel is $4.2 \AA$ in the STW-Si, and $4.7 \AA$ for STW-SiGe, which matches the medium-sized peaks of each PSD. The largest peak (I) represents the widest section of the pore, with a larger diameter than the largest diffusing particle. As the red area indicates in Figure 2, this peak corresponds to curved channels that connect other sections of the pore. The medium-sized peak (II) limits the diffusion of particles along the chiral channel of STW and, as seen in the blue area of Figure 2, is a neck between the curved channels (I). The leftmost peak (III) is located in each distribution at a lower diameter than the largest diffusing particle for each case. This section of the pore, as it does not limit the diffusion of particles along the chiral channel, can be described as an elongation of the curved channels (I) with the shape of a pocket (III). For clarity, the different pore sections of the pore will be referred to as curved channels (I), necks (II) and pockets (III) throughout the manuscript. The contribution of these pore sections builds the chiral channel and cages characterizing the topology of STW (grey silhouette of Figure 2). Although both structures are very similar, the chiral channel in STW-SiGe is significantly larger and the proportional contribution of necks to the accessible volume is smaller (II).

The adsorption mechanisms of three structural isomers of pentanol $\left(\mathrm{C}_{5} \mathrm{H}_{12} \mathrm{O}\right)$ in the frameworks STW-Si and STW-SiGe are investigated first. Since the force field used in this work has been derived by combining different parametrizations, the consistency of the overall force field had to be established. For this purpose, adsorption isotherms for 1-, 2- and 3-pentanol (1P, 2P, 3P) were computed in STW-Si at $313 \mathrm{~K}$ by CFCMC simulations. The resulting data are plotted in Figure 3 along with experimental data obtained according to the experimental section. Considering that no adjustment was required, the agreement proves that the calculated host-guest interactions are reliable. Next, the adsorption isotherms of the $R$ and $S$ enantiomers of $2 \mathrm{P}, 2 \mathrm{MB}$, and $3 \mathrm{M} 2 \mathrm{~B}$ as pure compounds are studied in both structures at $298 \mathrm{~K}$ (Figure 4). Given the size of the channels of STW-Si and STW-SiGe, mean square displacements (MSD) were calculated by Molecular Dynamics simulations to check if the compounds can diffuse inside the frameworks (Figure S2 of the Supporting Information). Diffusion coefficients for $R$ - and $S-2 \mathrm{P}$ are one order of magnitude higher for STW-SiGe $\left(10^{-10} \mathrm{~m}^{2} \mathrm{~s}^{-1}\right)$ than for STW-Si $\left(10^{-11} \mathrm{~m}^{2} \mathrm{~s}^{-}\right.$ $\left.{ }^{1}\right)$. Diffusion coefficients for $2 \mathrm{MB}$ enantiomers in STW-SiGe are around $10^{-10} \mathrm{~m}^{2} \mathrm{~s}^{-1}$ and they do not reach the diffusive regime in STW-Si. Likewise, 3M2B only diffuses in STW-SiGe, with diffusion coefficients of $10^{-11} \mathrm{~m}^{2} \mathrm{~s}^{-1}$ for both $R$ and $S$ enantiomers. Thus, the chiral channel in STW-SiGe, which is $0.4 \AA$ wider than the channel in STW-Si, allows diffusion of all compounds, while only 2P diffuses in STW-Si. Consequently, in the all-silica structure we focus on the adsorption isotherms of $R$ - and $S-2 \mathrm{P}$.

Overall, the isotherms show similar shape with a steep slope evidencing the rapid pore filling usual in adsorption of alcohols ${ }^{38,39}$. The onset pressure of adsorption can be set at $1 \mathrm{~Pa}$ for $2 \mathrm{P}, 10 \mathrm{~Pa}$ for $2 \mathrm{MB}$ and $100 \mathrm{~Pa}$ for $3 \mathrm{M} 2 \mathrm{~B}$. At saturation, $2 \mathrm{P}$ reaches about 6.6 and 8.7 molecules per unit cell in STW-Si and STW-SiGe, respectively. Saturation in STW-SiGe is reached with about $7.6(2 \mathrm{MB})$ and $7(3 \mathrm{M} 2 \mathrm{~B})$ molecules per unit cell. In general terms, differences in the adsorption values between $R$ and $S$ enantiomers of each pair stay within the error bars. Nevertheless, there are clear differences in the adsorption of $R$ - and $S$-3M2B. In the low-coverage regime, the value of the $S$ enantiomer is twice the value of $R$, and, at saturation, $S$ enantiomer packs better than $R$ and it allows one additional molecule in the structure. The adsorption of $2 \mathrm{P}$ is higher in STW-SiGe than in STW-Si as expected for the higher volume and wider pore size of the former framework. The increasing adsorption of $3 \mathrm{M} 2 \mathrm{~B}, 2 \mathrm{MB}$ and $2 \mathrm{P}$ in STW-SiGe indicates that linear isomers pack more efficiently in the structure than branched isomers.

The adsorption of the molecules in the flexible and rigid structures is shown in Figure 4. Differences between values of adsorption using the rigid and flexible frameworks lie within the error tolerance and validate the force field. Note that the adsorption in the saturation regime also reaches similar values 

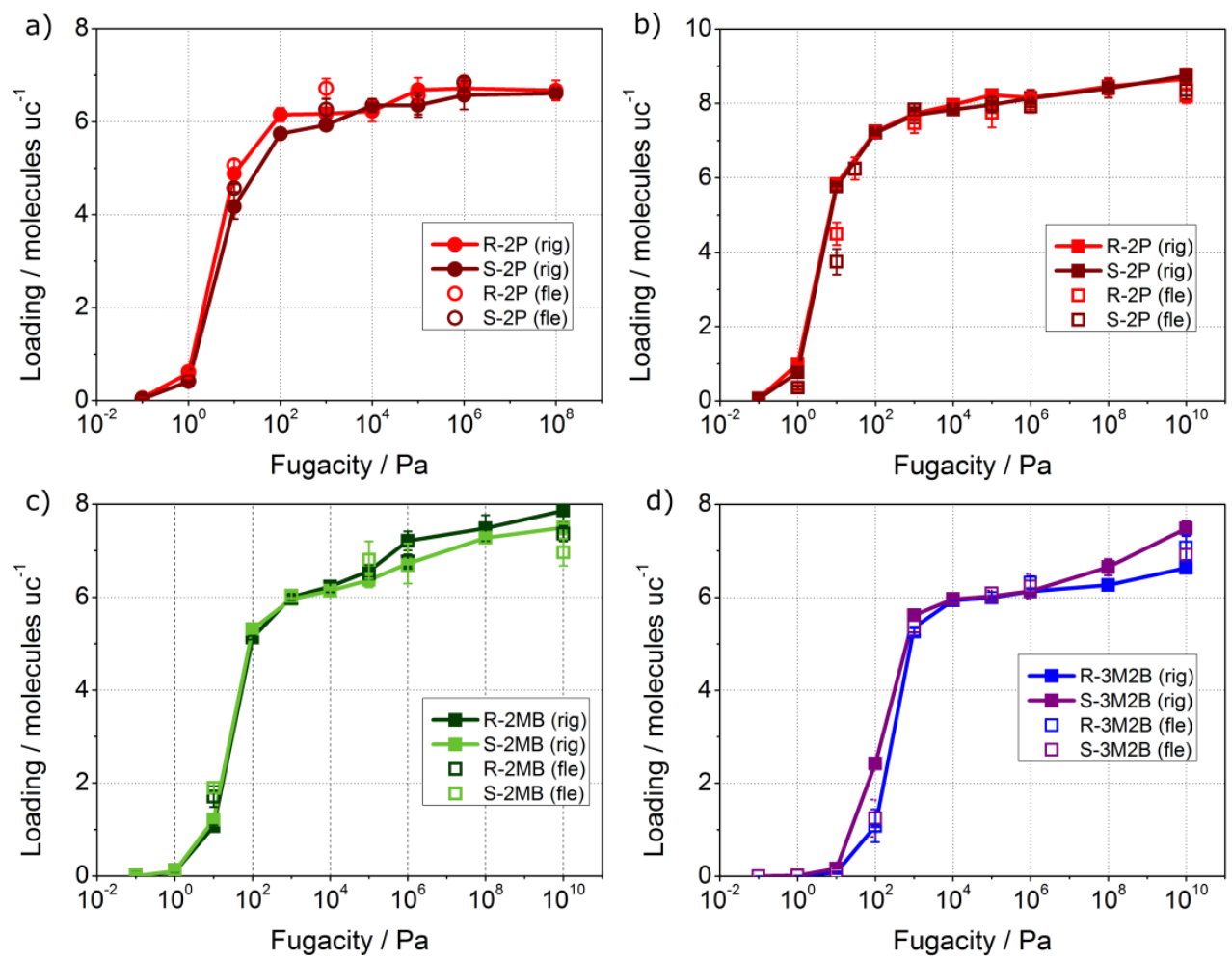

Figure 4. Single-component adsorption isotherms (298K) of enantiomeric pairs of a) $2 \mathrm{P}$ in STW-Si, b) $2 \mathrm{P}$ in $\mathrm{STW}-\mathrm{SiGe}$, c) $2 \mathrm{MB}$ in STW-SiGe, and d) $3 \mathrm{M}_{2} \mathrm{~B}$ in STW-SiGe, modelled as rigid (full symbols) and flexible frameworks (open symbols).

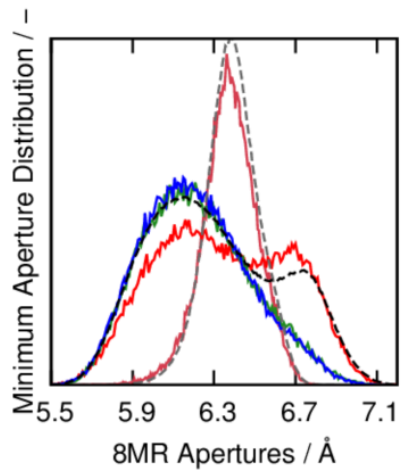

Si 2P-R 6.85

SiGe 2P-R 8.07 SiGe 2MB-R 7.36

SiGe 3M2B-R 7.07

Si empty

Gempty ---.--

Si $2 P-R \quad 6.85$

SiGe 2P-R 8.07

SiGe 2MB-R 7.36

SiGe 3M2B-R 7.07

Si empty --.-...

SiGe empty

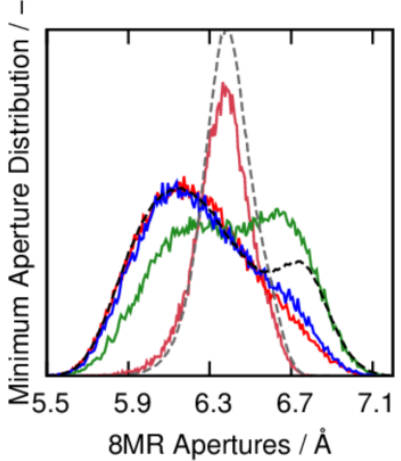

Si 2P-S 6.84 SiGe 2P-S 7.90 SiGe 2MB-S 6.96

SiGe 3M2B-S 6.91

Si empty

SiGe empty ------
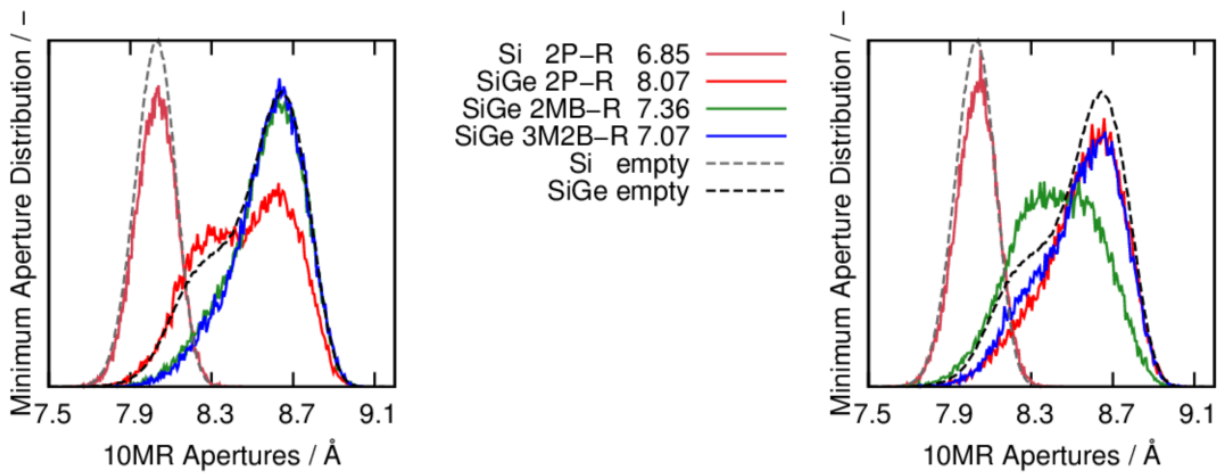

Si 2P-S 6.84

SiGe 2P-S 7.90

SiGe 2MB-S 6.96

SiGe 3M2B-S 6.91

Si empty ------

SiGe empty

10MR Apertures / $\AA$

Figure 5. Distribution of minimum apertures of 8MR (top) and 1oMR (bottom) in the empty STW-Si and STW-SiGe frameworks (dashed lines), and saturated with molecules (solid lines). Loading is indicated for each compound in molecules per unit cell. 

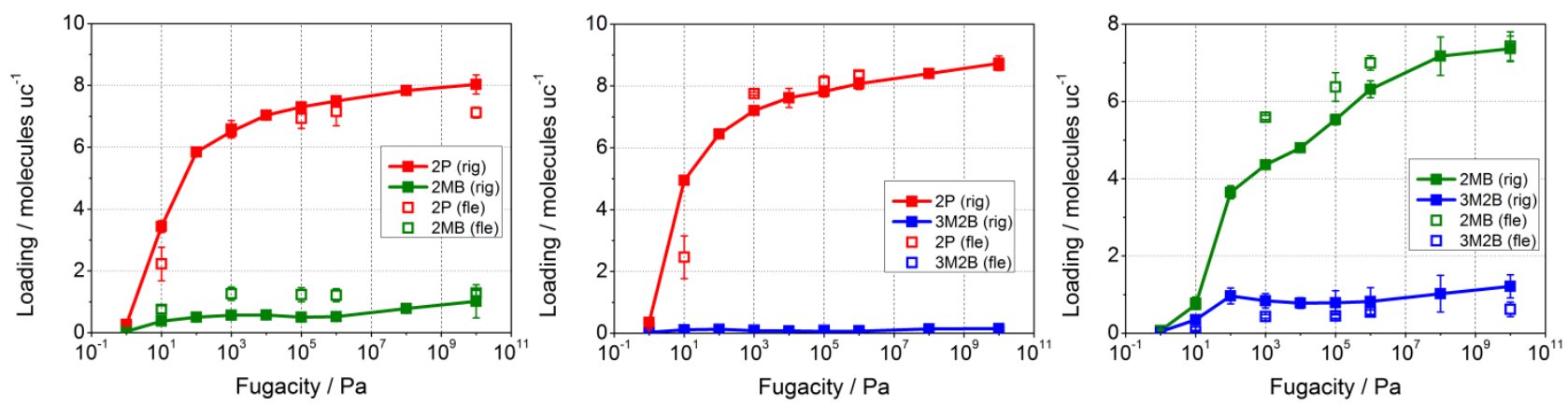

Figure 6. Adsorption isotherms of the equimolar mixtures of $2 \mathrm{P}-2 \mathrm{MB}$ (left), $2 \mathrm{P}-3 \mathrm{M} 2 \mathrm{~B}$ (center), and $2 \mathrm{MB}-3 \mathrm{M} 2 \mathrm{~B}$ (right) at $298 \mathrm{~K}$ in STW-SiGe, calculated with rigid and flexible frameworks.

i.e. the adsorption capacity of the structures is not altered by the flexibility of the lattice. However, in the case of $3 \mathrm{M} 2 \mathrm{~B}$ both enantiomers reach the same saturation values in the flexible structure, but not in the rigid framework. We also found some differences in the low-coverage regime. The adsorption of $2 \mathrm{P}$ (in $\mathrm{STW}-\mathrm{Si}$ ) and $2 \mathrm{MB}$ (in STW-SiGe) at $10 \mathrm{~Pa}$ is slightly larger in the flexible framework for both pairs of enantiomers, while the adsorption of $R / S-2 \mathrm{P}$ (at $10 \mathrm{~Pa}$ ) and $R / S-3 \mathrm{M} 2 \mathrm{~B}$ (at $100 \mathrm{~Pa}$ ) in the flexible STW-SiGe is lower than in the rigid framework. The adsorption of $R$ and $S$ enantiomers of 3M2B in the low-coverage regime follows the same trend than at saturation. The value of adsorption of $S-3 \mathrm{M} 2 \mathrm{~B}$ in the flexible framework compared to the rigid one decreases to the same value than $R-3 \mathrm{M} 2 \mathrm{~B}$, reducing the $R / S$ difference in the adsorption which apparently reflects no selectivity. This behavior upon introducing flexibility in the framework model suggests some coupling between the thermal lattice vibration and the adsorbates.

To analyze the changes that frameworks undergo during adsorption we calculated the minimum aperture of 8- and 10membered rings, i.e., the shortest distance between opposite oxygen atoms. These calculations were performed from snapshots generated during the hybrid MCMD simulations. The resulting values were time averaged for each compound, and compared to the values of minimum aperture calculated for the empty frameworks. The resulting histogram is plotted in Figure 5. The empty STW-SiGe framework used as a reference shows a broad peak with maxima at 6.1 and $6.75 \AA$ in the 8 membered rings $(8 \mathrm{MR})$, whereas the 10 -membered rings (10MR) show a maximum at $8.61 \AA$ and a shoulder at $8.3 \AA$. This suggests a non-trivial geometric coupling between the 8MR and 10MR: the former shrinks even below the STW-Si level while the latter broadens. The distribution of window apertures changes for all components when molecules saturate the structure, which points to a host-guest coupling in the flexible STW-SiGe framework. However, given that the adsorption of different compounds leads to different distributions, it can be inferred that the widening or shrinkage of window apertures must be related to the adsorption sites and the microassembly of molecules, depending on the adsorbate. In general terms, upon alcohol adsorption the aperture of $8 \mathrm{MR}$ tends to a smaller size and narrower distribution while that in $10 \mathrm{MR}$ tends to a wider size and narrower distribution, effectively constraining the aperture and evidencing the geometric coupling between 8 and 10MR. A noticeable exception is $S-2 \mathrm{MB}$ that broadens the distribution and makes it less specific than in the empty framework. Contrary to this, the STW-Si framework shows one single peak at $6.4 \AA$ and $8 \AA$, for the $8 \mathrm{MR}$ and $10 \mathrm{MR}$, respectively. Thus, the thermal lattice vibration of STW-Si is not coupled to the sorbate molecules, since the defined peaks remain unaffected by the different loadings and sorbate molecules.

The channel system of STW-Si and STW-SiGe suggests possible applications as molecular sieves for compounds with different structural features. Thus, we study the selectivity of these frameworks towards structural isomers. Moreover, the fact that $2 \mathrm{P}$ is adsorbed in STW-SiGe at lower pressure values than $2 \mathrm{MB}$ and $3 \mathrm{M} 2 \mathrm{~B}$, points to a preferential adsorption of the linear isomer in this structure. To corroborate this selective behavior, adsorption isotherms of equimolar binary mixtures of racemic 2P-2MB, 2P-3M2B and 2MB-3M2B in STW-SiGe at $298 \mathrm{~K}$ are computed and shown in Figure 6. The adsorption isotherms reveal that STW-SiGe strongly favors the adsorp tion of $2 \mathrm{P}$ over $2 \mathrm{MB}$ and $3 \mathrm{M} 2 \mathrm{~B}$, as expected, and of $2 \mathrm{MB}$ over $3 \mathrm{M} 2 \mathrm{~B}$. In the mixtures of $2 \mathrm{P}-2 \mathrm{MB}$ and $2 \mathrm{MB}-3 \mathrm{M} 2 \mathrm{~B}$, the least adsorbed compound reaches at most around 1 molecule per unit cell, while the favored compound reaches around 8 molecules per unit cell. More interestingly, in the mixture of $2 \mathrm{P}$ and $3 \mathrm{M} 2 \mathrm{~B}$, the linear isomer fills the structure completely, and leaves no useful space in the channel for $3 \mathrm{M} 2 \mathrm{~B}$ to be adsorbed. The trend in the preferential adsorption from $2 \mathrm{P}$ to $3 \mathrm{M} 2 \mathrm{~B}$ follows a similar pattern than that established in previous studies ${ }^{37-39}$, where a linear molecule is preferred over mono or dibranched molecules. According to this pattern, $2 \mathrm{P}$ is the most linear molecule and is preferentially adsorbed in the channel. Contrary to this, $2 \mathrm{MB}$ is topologically a monobranched alcohol with a methyl group very close to its center of mass. Finally, 3M2B is a dibranched alcohol which makes it especially bulky and the least favored in terms of preferential adsorption. This selective behavior of STW-SiGe holds true both in the rigid and flexible model of the framework. Still, as in the case of the pure systems, the adsorption in the flexible STW-SiGe shows some differences. Firstly, discrepancies in the filling behavior are consistent with those observed in the pure systems. Next, in the mixture of $2 \mathrm{P}-2 \mathrm{MB}$, adsorption values of $2 \mathrm{P}$ are slightly lower in the flexible framework, while those for $2 \mathrm{MB}$ are higher, yet these differences are no greater than 1 molecule per unit cell. Finally, in the third mixture, $2 \mathrm{MB}$ and $3 \mathrm{M} 2 \mathrm{~B}$, the adsorption of $3 \mathrm{M} 2 \mathrm{~B}$ reaches values below 1 molecule per unit cell, and $2 \mathrm{MB}$ reaches higher values, although at saturation they are equal to those of the rigid structure. Given that the topology of this 

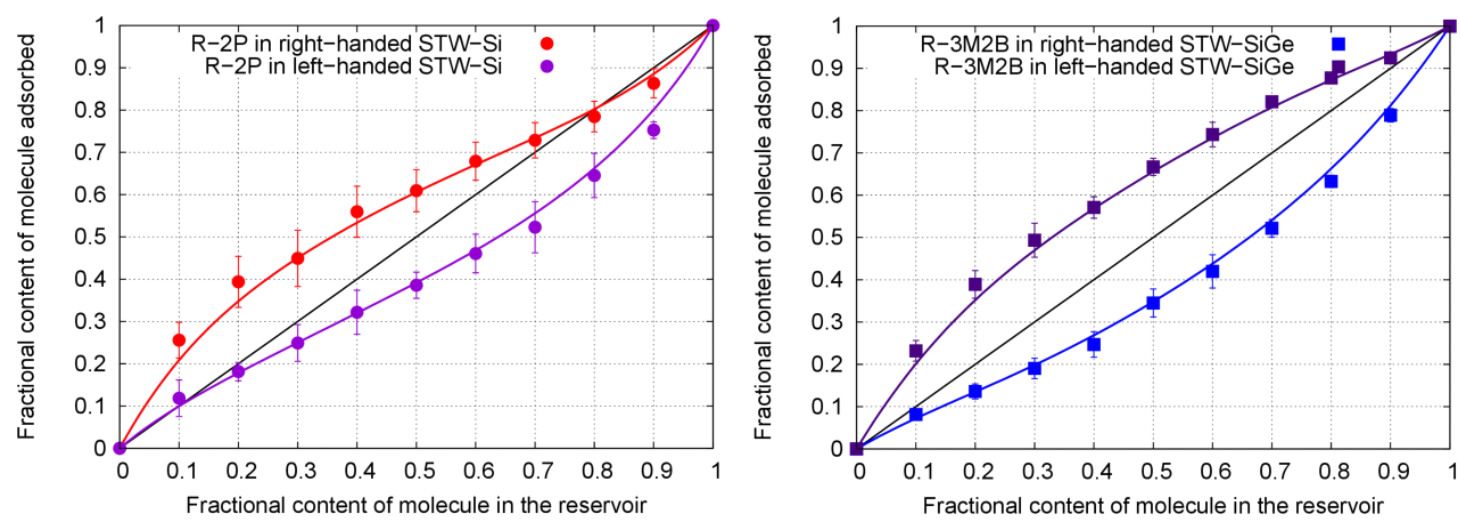

Figure 7. Adsorbed fractional content of $R$ enantiomers as a function of the $R$-fraction in a R/S mixture in the reservoir for right and left-handed STW-Si (left) and STW-SiGe (right) taken as rigid frameworks, at $10^{6} \mathrm{~Pa}$ and $298 \mathrm{~K}$. Straight line indicates that the adsorbed composition is identical to that in the reservoir. Solid line shows an approximation of the data trend using the Bezier curve smoothing.

framework is based on a helicoidal channel accessible to all molecules, structural selectivity is mainly a result of the preferential adsorption. It is worth considering that the coupling between lattice vibration and sorbate depends on the uptake and sorbate type. Thus, most of the differences observed are connected to the wider amplitude of vibrations of STW-SiGe framework seen in Figure 5. This may create variable sites into which a new molecule fits leading to subtle changes in the structural selectivity (Figure 6). Specifically, the different trends that window apertures show for $2 \mathrm{MB}$ and $3 \mathrm{M} 2 \mathrm{~B}$ and the competition of each component for adsorption sites generate adsorption values that are not predicted when the framework is modelled as rigid. However, the trends in preferential adsorption remain, and the selectivity for each mixture is at least 5.

Another exciting aspect addressed in this work is the effect of flexibility in the enantioselectivity of STW-Si and STWSiGe. For this purpose, enantioselectivity is analyzed first for the rigid frameworks. Figure 7 shows the fractional content of the $R$ enantiomer in the adsorbed phase against the fractional content of the same enantiomer set in the bulk at conditions of $10^{6} \mathrm{~Pa}$ and $298 \mathrm{~K}$. The straight line indicates a lack of enantioselectivity, i.e., values of fractional content in the adsorbed phase equal those in the bulk. For 2P in STW-Si and 3M2B in STW-SiGe, a significant deviation from the straight line is seen in Figure 7, while $2 \mathrm{P}$ and $2 \mathrm{MB}$ in STW-SiGe remain close to the straight line as is shown in Figure S3 of the Supporting Information. In the case of 2P in STW-Si the fractional content of the $R$ enantiomer is higher than that set in the bulk below 0.7 , and falls on top of the straight line above 0.8 . This indicates that right-handed STW-Si is selective towards the $R$ $2 \mathrm{P}$ enantiomer while the fractional content is below 0.7 . On the contrary, the fractional content of $R-3 \mathrm{M} 2 \mathrm{~B}$ in right-handed STW-SiGe is lower than that in the bulk for all compositions. This reveals that right-handed STW-SiGe is selective towards $S$-3M2B.

The handedness of the framework is irrelevant to structural separations and therefore the results obtained in previous section were obtained from the right-handed frameworks of STW-Si and STW-SiGe. However, enantioselectivity is affected in a predictable way by the handedness of the framework. Figure 7 shows the curves obtained with GCMC simula- tions using the right and left-handed frameworks. As expected, the curves are inverted around the central point $(0.5,0.5)$. Then, the left-handed STW-Si enriches the mixture in $S-2 \mathrm{P}$ instead of $R-2 \mathrm{P}$, following the same behavior when the fractional content of the preferred enantiomer increases. The lefthanded STW-SiGe adsorbs preferentially the opposite enantiomer, $R-3 \mathrm{M} 2 \mathrm{~B}$. This inversion in adsorption selectivity is expected since opposite enantioselectivity in enantiomeric frameworks has been reported previously ${ }^{13,17}$. However, it has not been analyzed yet on scalemic mixtures and, to the best of our knowledge, this almost exact symmetry in the chiral adsorption has not been shown before. It also shows in an independent analysis from our error bars that the uncertainties on the data are small given that the simulations are independent from each other.

The previously explained results clarify the complex relation between confinement and enantioselectivity. Even though STW-Si and STW-SiGe have the same topology, the $0.4 \AA$ wider pores of the latter are enough to disrupt the chiral selectivity towards $R-2 \mathrm{P}$ in STW-SiGe, whereas STW-Si does display chiral resolution. Thus, $2 \mathrm{P}$ fits tightly enough in the chiral channel of STW-Si, but both enantiomers can move and adsorb more freely in the wider channel of STW-SiGe. Indeed, STW-SiGe is not selective for $2 \mathrm{MB}$ either, yet it is for $3 \mathrm{M} 2 \mathrm{~B}$. As mentioned in the analysis of the structural adsorption, $3 \mathrm{M} 2 \mathrm{~B}$ is the bulkiest molecule among these three compounds. Consequently, the chiral recognition of the $S$ enantiomers of 3M2B happens only in STW-SiGe, in whose channels the molecules of $3 \mathrm{M} 2 \mathrm{~B}$ fit tightly.

Once the chiral selectivity of the structures under study had been understood in the rigid frameworks, we investigated how this selectivity is affected by lattice vibrations. To this end, we performed hybrid MCMD simulations in which framework atoms can move. The obtained results are compared to the values obtained for the rigid model using energetically optimized frameworks. As shown in Figure 8 the flexible STW-Si framework maintains the enantioselectivity towards $R-2 \mathrm{P}$. Minor variations with respect to the rigid structure are observed and fall within the error bars. In the case of the flexible STW-SiGe framework, the observed preference towards $S$ $3 \mathrm{M} 2 \mathrm{~B}$ decreases strongly and the values of fractional content of this enantiomer in the framework reach the straight line, 

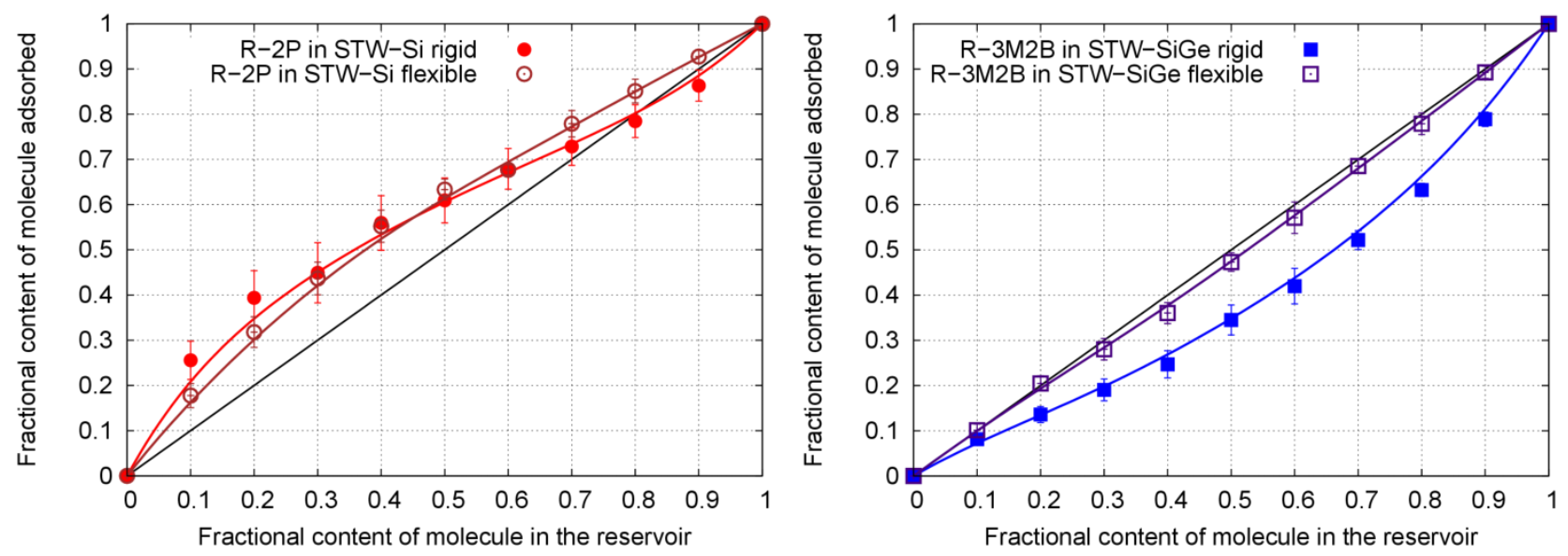

Figure 8. Adsorbed fractional content of $R$ enantiomers as a function of the $R$-fraction in a $R / S$ mixture in the reservoir for right and left-handed STW-Si (left) and STW-SiGe (right) taken as flexible (open symbols) and rigid (full symbols) frameworks, at $10^{6}$ $\mathrm{Pa}$ and $298 \mathrm{~K}$. The straight line indicates that the adsorbed composition is identical to that in the reservoir. Solid lines capture the data trend by using Bezier curves smoothing.

indicating no selectivity. In terms of enantiomeric excess $(\%$ ee), it decreases from $31 \pm 7 \%$ in the rigid model for the framework of STW-SiGe to $5 \pm 4 \%$ when the framework atoms are allowed to move at $298 \mathrm{~K}$. Despite this, in the case of STW-Si the vibration of the lattice not only does retain its chiral selectivity but it also increases up to $26 \pm 5 \%$ while the $e e$ in the rigid framework is $22 \pm 10 \%$.

We performed additional GCMC simulations to ensure that differences in the enantioselective behavior are not a consequence of a lack of efficiency of the insertion method during the hybrid MCMD simulations. The fractional content of $R$ enantiomers in the adsorbed phase from a racemic feed has been calculated by averaging results from 34 individual simulations based on snapshots of STW-Si and STW-SiGe frameworks taken from the most stable framework configurations during the hybrid MCMD simulations performed to compute enantioselectivity and freezing the framework atoms. The average $R$-fraction obtained is $0.622( \pm 0.045)$ and $0.489( \pm$ 0.033 ) for the snapshots of STW-Si and STW-SiGe, respectively. This indicates that the lattice vibrations in STW-Si generate intermediate frameworks that are still enantioselective, whereas the intermediate frameworks of STW-SiGe show an average that is barely enantioselective.

In the very recent report by Brand et al, the single component adsorption of 2-butanol on enantiomerically enriched STW-SiGe suggested a moderate enantioselectivity for $R$ and $S$ enantiomers on the right and left enriched zeolites, respectively, i. e., the same hand preference as calculated by us for 2pentanol. The adsorbents were significantly enriched in $\mathrm{Si}$ compared to $\mathrm{Ge}$ ( $\mathrm{Si} / \mathrm{Ge}$ in the gels were 2 or above), so their enantioselectivity could be larger than the one we calculate for the materials with $\mathrm{Si} / \mathrm{Ge}=1$. Our findings suggest, however, that a significantly larger selectivity could be achieved by the pure silica zeolite. Considering the larger thermal and hydrothermal stability of pure silica zeolites, that material could also avoid the low adsorption values obtained by Brand et al. with materials with low crystallinity (a factor of 4 between the butanol uptakes in right and left handed materials was considered due to differences in crystallinity).
The basic idea that is extracted from the analysis of the chiral selectivity in STW-Si and STW-SiGe is that the intrinsic flexibility of zeolites, that is, their natural lattice vibrations, may disrupt the enantioselectivity of the framework, though not always. To understand how the framework is changing in such a way that it can alter its preferential adsorption, PSDs have been calculated for the intermediate frameworks and compared to the obtained from a MD simulation without sorbates (Figure 9). The corresponding window apertures calculated from snapshots generated during the related hybrid MCMD simulations are shown in Figure S4 in the Supporting Information. The window aperture of 8MR and 10MR of STW-Si in Figure S4 remains as invariable as in Figure 5. Their counterparts in STW-SiGe saturated with 3M2B show one wide peak at $6.15 \AA$ and another at $8.61 \AA$, corresponding to the narrow sized 8MR and the large sized 10MR, respectively. Regarding the PSDs (Figure 9), the peaks are well separated in the rigid frameworks but in the flexible structures the distribution of pore space is broad. This explains the greater structural disorder and greater variety of adsorption sites found for the flexible framework. More precisely, the STW-Si framework maintains two peaks, corresponding to the curved channels $(4.71 \AA)$ and necks connecting channels $(4.41 \AA)$ while the leftmost peak corresponding to the pockets disappears. This is true even in the empty, flexible framework (Figure 9). In the STW-SiGe framework, the leftmost, large peak disappears too, and the central peak, which is of low intensity in the rigid framework, blends with the largest-pore peak, corresponding to the curved channels (5.13 $\AA$ ). However, in the empty, flexible framework of STW-SiGe the largest-pore peak is slightly displaced to the left $(5.05 \AA)$ and a small shoulder arises (4.49 $\AA$ ) at a position between the central and small- pore peaks of the rigid framework. This difference is comparable to that observed in the distribution of window apertures for empty and saturated silicogermanate frameworks (Figures 5 and S4), and consistent with the host-guest coupling enabled by the flexibility of the framework. Thus, the intrinsic flexibility of the all-silica framework is rather limited and largely sorbateindependent. On the contrary, the flexibility of the 

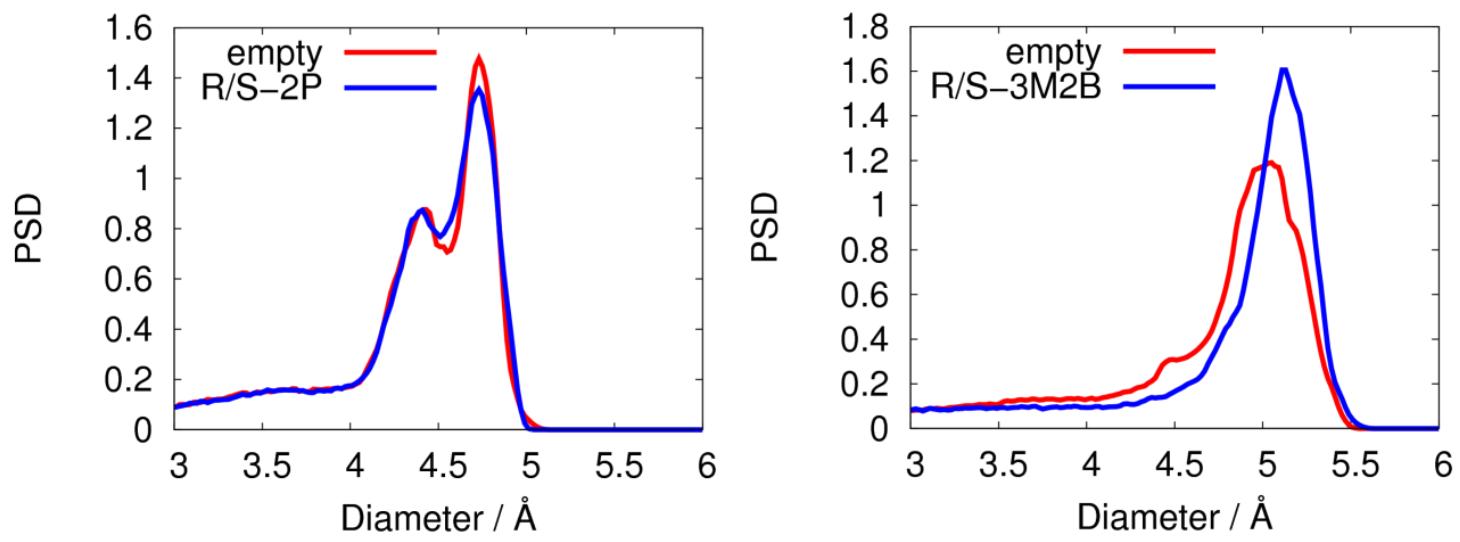

Figure 9. Pore size distribution of flexible STW-Si (left) and STW-SiGe (right) averaged from snapshots of frameworks taken during MD simulations of the empty frameworks (red) and hybrid MCMD simulations from a racemic feed (blue).

silicogermanate framework is large and sorbate-dependent, and responsible for the loss of well-defined pore spaces. In addition, the shrinkage of $8 \mathrm{MR}$ is related to the smoothing of the cage surface which, coupled to the aperture of 10MR, leads to a loss of adsorption restrictions and, therefore, a loss of selectivity.

The last question is how the change in the pore size distribution is causally related to the selective adsorptions of enantiomers of 3M2B in STW-SiGe and 2P in STW-Si. The loss of selectivity in a model structure in which smoothing out of the internal pore space is observed strongly suggests that the geometry of the chiral channel rather than guest-guest interactions is responsible for chiral separation in these systems. In STW-Si, only the smallest pore sections (necks and pockets) are slightly smoothed and chiral separation for $2 \mathrm{P}$ is retained, whereas in STW-SiGe, the pore space smoothing is large and chiral separation for 3M2B is lost. To understand this in detail, selectivity was studied depending on loading (Figure S5 in the Supporting Information). These results were obtained by averaging five independent GCMC simulations from a racemic feed in STW-Si and STW-SiGe rigid frameworks selected from the intermediate frameworks used previously. It can be observed that from 1 molecule per unit cell up to 7, the $R$ fraction remains nearly constant. Indeed, $R$-fraction values of $2 \mathrm{P}$ are similar and constant in the rigid minimized STW-Si and its intermediate frameworks. In the rigid STW-SiGe, the $R$ fraction of $3 \mathrm{M} 2 \mathrm{~B}$ remains similar while increasing the loading. Standing for what has been already observed, the $R$ fraction of $3 \mathrm{M} 2 \mathrm{~B}$ averaged over the intermediate frameworks of STW-SiGe is constant but close to 0.5 , as STW-SiGe is not enantioselective. Given this, it might be interesting to understand how the adsorption sites in the structure relate to the PSD. The average occupational density profiles found in Figures S6 and S7 in the Supporting Information show the preferential sites of adsorption for each of the molecules under study. Specifically, $2 \mathrm{P}$ is mostly present in the curved channels, whereas $3 \mathrm{M} 2 \mathrm{~B}$ is especially dense in the pockets that arise from the curved channels. Thus, since the widest peak in the PSD of STW-Si, corresponding to the curved channels, is kept, the adsorption site where the chiral recognition of $2 \mathrm{P}$ happens is also kept. In the case of STW-SiGe, the peak corresponding to the pockets, where $3 \mathrm{M} 2 \mathrm{~B}$ is preferentially adsorbed, is lost. This is caused by the smoothing of the cage, because of the shrinkage of the $8 \mathrm{MR}$ in STW-SiGe. Thus, the adsorption site where the chiral recognition of $3 \mathrm{M} 2 \mathrm{~B}$ occurs is disrupted. In fact, this can be observed at a lesser degree in the single-component adsorption isotherm of 3M2B (Figure 4). In the rigid STW-SiGe slight differences in the adsorption of $R$ and $S-3 \mathrm{M} 2 \mathrm{~B}$ could be seen at low-loading regime and at saturation. In the flexible STW-SiGe the chiral recognition site of $3 \mathrm{M} 2 \mathrm{~B}$ is disrupted and the previously differences observed in the adsorption converge to the same values. Then, although the molecule can be adsorbed, its $S$ enantiomer cannot be discriminated from the $R$ enantiomer.

\section{Conclusions}

The adsorption of $2 \mathrm{P}, 2 \mathrm{MB}$, and $3 \mathrm{M} 2 \mathrm{~B}$ was studied in the all-silica and silicogermanate frameworks of STW zeolite. The theoretical methods are validated by a reasonable match of the calculated and experimental adsorption isotherms.

Adsorption was calculated assuming first rigid frameworks. In terms of structural separation, STW-Si separates the linear from the branched isomers since the latter cannot enter the framework. The silicogermanate structure shows preferential adsorption for $2 \mathrm{P}$ over $2 \mathrm{MB}$ and $3 \mathrm{M} 2 \mathrm{~B}$, and for $2 \mathrm{MB}$ over $3 \mathrm{M} 2 \mathrm{~B}$. This preferential adsorption is attributed to the efficiency of the molecular arrangement that increases from $3 \mathrm{M} 2 \mathrm{~B}$ to $2 \mathrm{P}$. Regarding chiral selectivity the right handed STW-Si is selective towards $R-2 \mathrm{P}$, while right-handed STW$\mathrm{SiGe}$ is selective towards $S$-3M2B. This enantioselectivity is driven by the geometry of the chiral channel and, therefore, chiral recognition is mainly due to confinement.

The introduction of NVE MD simulations into a GCMC scheme revealed a coupling between adsorbates and lattice vibration in the case of STW-SiGe which depends on the sorbate. However, the intrinsic flexibility of the STW-Si is independent of the type of sorbate and loading or its absence. The wide distribution of window apertures in the silicogermanate framework affects its internal apertures size distribution and leads to differences in the separation performance. In general terms, the adsorption calculated using the rigid and flexible models matches and we found this structure suitable for the separation of the structural isomers, independently of the model used.

Chiral selectivity is altered when the intrinsic flexibility of the zeolite that causes structural changes affects the chiral 
recognition sites. The static lattice vibration around the equilibrium position of the all-silica framework of right-handed STW smooths the surface of the structure but keeps the adsorption sites that select $R-2 \mathrm{P}$ over $S-2 \mathrm{P}$. Lattice vibration is larger for STW-SiGe due to the presence of germanium atoms. This distorts the pore size distribution of the structure and results on the loss of selectivity by altering the chiral recognition site for 3M2B in STW-SiGe. These results suggest an enantioenriched pure silica STW should show a better enantioselectivity than the silicogermanates recently reported.

The increasing degree of influence that intrinsic flexibility might have in selective behaviors leads to the question of how to approach these issues computationally. Although computing flexibility for the systems under study is currently effective and could provide a more realistic view of the further separation process, it is still very expensive in terms of computational cost. Thus, regardless of modelling the structure as rigid or flexible, it is interesting to consider the possible effect of lattice vibrations in selectivity by focusing on structural distortions in the lattice, the possible host-guest coupling and how it might affect the molecular mechanism of separation. In fact, as this work has proven, reliable predictions of the suitability of a given structure for the separation of similar compounds, such as enantiomers, are difficult to obtain if its flexibility is not considered appropriately, even though this flexibility, like for STW, is assumed to be limited to lattice vibration.

\section{ASSOCIATED CONTENT}

Supporting Information. Detailed information of force fields parameters, MSDs plot in the rigid frameworks; adsorbed fractional content of $R-2 \mathrm{P}$ and $R-2 \mathrm{MB}$ in rigid and flexible STW-SiGe; distribution of minimum apertures in empty STW-Si and STW-SiGe frameworks, and saturated with racemic mixtures; adsorbed fractional content as a function of loading; average occupational density profiles. "This material is available free of charge via the Internet at http://pubs.acs.org."

\section{AUTHOR INFORMATION}

\section{Corresponding Author}

* Sofia Calero: scalero@upo.es

*Patrick J. Merkling: pjmerx@upo.es

\section{Author Contributions}

All authors have given approval to the final version of the manuscript.

\section{Funding Sources}

This work was supported by the Spanish Ministerio de Economía y Competitividad (CTP2016-80206-P and MAT2015-71117-R) and the National Creative Research Initiative Program (2012R1A3A-2048833) through the National Research Foundation of Korea. S. R. G. Balestra thanks the Spanish MINECO for his predoctoral fellowship (BES-2014067825).

\section{ACKNOWLEDGEMENT}

We thank A. R. Ruiz-Salvador for his useful discussions about the generation of realistic distributions of silicon and germanium atoms in the structures and J. M. Vicent-Luna for implementing a new tool to plot average density profiles more efficiently.

\section{ABBREVIATIONS}

MOF, Metal-Organic Framework; HPLC, High-Performance Liquid Chromatography; CSP, chiral stationary phase; MSD; mean-squared displacement; PSD, pore size distribution; CFCMC, Continuous Fractional Component Monte Carlo; GCMC, Grand Canonical Monte Carlo; MD, Molecular Dynamics; $1 \mathrm{P}$, 1-pentanol; $2 \mathrm{P}$, 2-pentanol; $3 \mathrm{P}$, 3-pentanol; $2 \mathrm{MB}$, 2-methylbutanol; 3M2B, 3-methyl-2-butanol.

\section{REFERENCES}

(1) Zhang, J.; Chen, S.; Wu, T.; Feng, P.; Bu, X., J. Am. Chem. Soc. 2008, 130 (39), 12882-12883.

(2) Smith, S. W., Toxicol. Sci. 2oo9, 110 (1), 4-30.

(3) Berger, R. G., Flavors E Fragrances: Chemistry, Bioprocessing and Sustainability. Springer: Germany, 2007.

(4) Hasaneen, M. N., Herbicides - Mechanisms and Mode of Action. InTech: 2011.

(5) Daiha, K. d. G.; Angeli, R.; Oliveira, S. D. d.; Almeida, R. V., PLOS One 2015.

(6) Sharma, R.; Chisti, Y.; Banerjee, U. C., Biotechnol. Adv. 2001, 19, 627-662.

(7) Blaser, H.-U.; Pfaltz, A.; Wennemers, H., Chiral compounds. In Ullmann's Encyclopedia of Industrial Chemistry, John Wiley and Sons: Online, 2012.

(8) Shen, J.; Okamoto, Y., Chem. Rev. 2016, 116, 1094-1138.

(9) Okamoto, Y.; Ikai, T., Chem. Soc. Rev. 20o8, 37 (12), 2593-2608.

(10) Kuang, X.; Ma, Y.; Su, H.; Zhang, J.; Dong, Y.-b.; Tang, B., Anal. Chem. 2014, 86 (2), 1277-1281.

(11) Zhang, M.; Pu, Z.-J.; Chen, X.-L.; Gong, X.-L.; Zhu, A.X.; Yuan, L.-M., Chem. Commun. 2013, 49 (49), 5201-5203.

(12) Jiang, J.; Babarao, R.; Hu, Z., Chem. Soc. Rev. 2o11, 40 (7), 3599-3612.

(13) Zhang, L.; Jiang, J., J. Membr. Sci. 2011, 367 (1-2), 63-70.

(14) Castillo, J. M.; Vlugt, T. J. H.; Dubbeldam, D.; Hamad, S.; Calero, S., J. Phys. Chem. C 2010, 114 (50), 22207-22213.

(15) Clark, L. A.; Chempath, S.; Snurr, R. Q., Langmuir 2005, 21 (6), 2267-2272.

(16) Avery, K. A.; Mann, R.; Norton, M.; Willock, D. J., Top. Catal. 2003, 25 (1-4), 89-102.

(17) Dryzun, C.; Mastai, Y.; Shvalb, A.; Avnir, D., J. Mater. Chem. 2009, 19 (14), 2062-2069.

(18) Bao, X.; Broadbelt, L. J.; Snurr, R. Q., Microporous Mesoporous Mater. 2012, 157, 118-123.

(19) Bao, X.; Broadbelt, L. J.; Snurr, R. Q., Phys. Chem. Chem. Phys. 2010, 12 (24), 6466-6473.

(20) Bao, X.; Broadbelt, L. J.; Snurr, R. Q., Mol. Simul. 2oo9, 35 (1-2), 50-59.

(21) Jo, D.; Hong, S. B.; Camblor, M. A., ACS Catal. 2015, 5 (4), $2270-2274$.

(22) Peng, Y.; Gong, T.; Cui, Y., Chem. Commun. 2013, 49 (74), 8253-8255.

(23) Xuan, W.; Ye, C.; Zhang, M.; Chen, Z.; Cui, Y., Chem. Sci. 2013, 4 (8), 3154-3159.

(24) Bonnefoy, J.; Legrand, A.; Quadrelli, E. A.; Canivet, J.; Farrusseng, D., J. Am. Chem. Soc. 2015, 137 (29), 9409-9416.

(25) Demuynck, A. L. W.; Goesten, M. G.; RamosFernandez, E. V.; Dusselier, M.; Vanderleyden, J.; Kapteijn, F.; Gascon, J.; Sels, B. F., ChemCatChem 2014, 6 (4), 2211-2214

(26) Canivet, J.; Farrusseng, D., RSC Adv. 2015, 5 (15), 112541125.

(27) Kumar, P.; Guliants, V. V., Microporous Mesoporous Mater. 2010, 132 (1-2), 1-14.

(28) Li, Y.; Yu, J.; Wang, Z.; Zhang, J.; Guo, M.; Xu, R., Chem. Mater. 2005, 17 (17), 4399-4405. 
(29) Yu, J.; Xu, R., J. Mater. Chem. 20o8, 18 (34), 4021-4021.

(30) Mazur, M.; Wheatley, P. S.; Navarro, M.; Roth, W. J.; Položij, M.; Mayoral, A.; Eliášová, P.; Nachtigall, P.; Čejka, J.; Morris, R. E., Nat. Chem. 2015, 8 (1), 58-62.

(31) Sun, J.; Bonneau, C.; Cantin, A.; Corma, A.; DíazCabañas, M. J.; Moliner, M.; Zhang, D.; Li, M.; Zou, X., Nature 2009, 458 (7242), 1154-1157.

(32) Tang, L.; Shi, L.; Bonneau, C.; Sun, J.; Yue, H.; Ojuva, A.; Lee, B.-L.; Kritikos, M.; Bell, R. G.; Bacsik, Z.; Mink, J.; Zou, X., Nat. Mater. 2008, 7 (5), 381-385.

(33) Rojas, A.; Arteaga, O.; Kahr, B.; Camblor, M. A., J. Am. Chem. Soc. 2013, 135 (32), 11975-11984.

(34) Rojas, A.; Camblor, M. A., Angewandte Chemie - International Edition 2012, 51 (16), 3854-3856.

(35) Ma, Y.; Oleynikov, P.; Terasaki, O., Nat. Mater. 2017, 16, 755-769.

(36) Brand, S. K.; Schmidt, J. E.; Deem, M. W.; Daeyaert, F.; Ma, Y.; Terasaki, O.; Orazov, M.; Davis, M. E., PNAS 2017, 114 (20), 5101-5106.

(37) Dubbeldam, D.; Krishna, R.; Calero, S.; Yazaydin, A. O., Angewandte Chemie - International Edition 2012, 51 (47), 1186711871.

(38) Bueno-Perez, R.; Merkling, P. J.; Gomez-Alvarez, P.; Calero, S., Chem. - Eur. J. 2017, 23 (4), 874-885.

(39) Bueno-Perez, R.; Gutierrez-Sevillano, J. J.; Dubbeldam, D.; Merkling, P. J.; Calero, S., ChemPhysChem 2015, 16 (13), 27352738.

(40) Bao, X.; Snurr, R. Q.; Broadbelt, L. J., Microporous Mesoporous Mater. 2013, 172, 44-50.

(41) Bao, X.; Snurr, R. Q.; Broadbelt, L. J., Langmuir 2009, 25 (18), 10730-10736.

(42) Moghadam, P. Z.; Düren, T., J. Phys. Chem. C 2012, 116, 20874-20881

(43) Martin-Calvo, A.; Calero, S.; Martens, J. A.; Van Erp, T. S., J. Phys. Chem. C 2014, 118 (27), 14991-14997.

(44) Caremans, T. P.; Van Erp, T. S.; Dubbeldam, D.; Castillo, J. M.; Martens, J. A.; Calero, S., Chem. Mater. 2010, 22 (16), 4591-4601.

(45) Schneemann, A.; Bon, V.; Schwedler, I.; Senkovska, I.; Kaskel, S.; Fischer, R. A., Chem. Soc. Rev. 2014, 43 (16), 6o626096.

(46) Llewellyn, P. L.; Bourrelly, S.; Serre, C.; Filinchuk, Y.; Ferey, G., Angewandte Chemie - International Edition 2006, 118, 7915-7918.

(47) Zhang, J.; Wu, H.; Emge, T. J.; Li, J., Chem. Comm. 2010, 46, 9152-9154.

(48) Mellot-Draznieks, C.; Serre, C.; Surble, S.; Audebrand, N.; Ferey, G., J. Am. Chem. Soc. 2005, 127 (46), 16273-16278.

(49) Gee, J. A.; Sholl, D. S., J. Phys. Chem. C 2016, 120, 370376.

(50) Fairen-Jimenez, D.; Moggach, S. A.; Wharmby, M. T.; Wright, P. A.; Parsons, S.; Düren, T., J. Am. Chem. Soc. 2011, 133 (23), 8900-8902.

(51) Balestra, S. R. G.; Bueno-Perez, R.; Hamad, S.; Dubbeldam, D.; Ruiz-Salvador, A. R.; Calero, S., Chem. Mater. 2016, 28 (22), 8296-8304.

(52) Kitaura, R.; Seki, K.; Akiyama, G.; Kitagawa, S., Angew. Chem., Int. Ed. 2003, 42 (4), 428-431.

(53) Sartbaeva, A.; Wells, S. A.; Treacy, M. M. J.; Thorpe, M. F., Nat. Mater. 2006, 5 (12), 962-965.

(54) Haldoupis, E.; Watanabe, T.; Nair, S.; Sholl, D. S., ChemPhysChem 2012, 13 (15), 3449-3452.

(55) Wu, J. Y.; Liu, Q. L.; Xiong, Y.; Zhu, A. M.; Chen, Y., J. Phys. Chem. B 2009, 113 (13), 4267-4274.

(56) Awati, R. V.; Ravikovitch, P. I.; Sholl, D. S., J. Phys. Chem. C 2013, 117, 13462-13473.
(57) Ghysels, A.; Moors, S. L. C.; Hemelsoet, K.; De Wispelaere, K.; Waroquier, M.; Sastre, G.; Van Speybroeck, V., J. Phys. Chem. C 2015, 119 (41), 23721-23734.

(58) Jose Gutierrez-Sevillano, J.; Calero, S.; Hamad, S.; Grau-Crespo, R.; Rey, F.; Valencia, S.; Palomino, M.; Balestra, S. R. G.; Rabdel Ruiz-Salvador, A., Chem. - Eur. J. 2016, 22 (29), 10036-10043.

(59) Vlugt, T. J. H.; Schenk, M., J. Phys. Chem. B 2002, 206, 12757-12763.

(6o) Zimmermann, N. E. R.; Jakobtorweihen, S.; Beerdsen, E.; Smit, B.; Keil, F. J., J. Phys. Chem. C 2007, 111 (46), 17370-17381.

(61) Patel, R. N., Adv. Appl. Microbiol. 2ooo, 47, 33-78.

(62) Kresse, G.; Furthmüller, J., Comput. Mater. Sci. 1996, 6 (1), 15-50.

(63) Kresse, G.; Furthmüller, J., Phys. Rev. B 1996, 54, 1116911186.

(64) Perdew, J. P.; Ruzsinszky, A.; Csonka, G. I.; Vydrov, O. A.; Scuseria, G. E.; Constantin, L. A.; Zhou, X.; Burke, K., Phys. Rev. Lett. 2008, 100 (13), 4

(65) Jorgensen, W. L.; Maxwell, D. S.; Tirado-Rives, J., J. Am. Chem. Soc. 1996, 118 (45), 11225-11236.

(66) Bai, P.; Tsapatsis, M.; Siepmann, J. I., J. Phys. Chem. C 2013, 117 (46), 24375-24387.

(67) Gould, T.; Bucko, T., J. Chem. Theory Comput. 2016, 12 (8), 3603-3613

(68) Hill, J. R.; Sauer, J., J. Phys. Chem. 1994, 98 (4), 12381244 .

(69) Dubbeldam, D.; Calero, S.; Ellis, D. E.; Snurr, R. Q., Mol. Simul. 2016, 42 (2), 81-101.

(70) Frenkel, D.; Smit, B., Understanding Molecular Simulation. From Algorithms to Applications. 2002.

(71) Shi, W.; Maginn, E. J., J. Chem. Theory Comput. 2007, 3 (4), 1451-1463.

(72) Dubbeldam, D.; Torres-Knoop, A.; Walton, K. S., Mol. Simul. 2013, 39 (14-15), 1253-1292.

(73) Balestra, S. R. G.; Hamad, S.; Ruiz-Salvador, A. R.; Domínguez-García, V.; Merkling, P. J.; Dubbeldam, D.; Calero, S., Chem. Mater. 2015, 27 (16), 5657-5667.

(74) Faller, R.; de Pablo, J. J., J. Chem. Phys. 2002, 116 (1), 5559.

(75) Ghoufi, A.; Subercaze, A.; Ma, Q.; Yot, P. G.; Ke, Y.; Puente-Orench, I.; Devic, T.; Guillerm, V.; Zhong, C.; Serre, C.; Ferey, G.; Maurin, G., J. Phys. Chem. C 2012, 116 (24), 13289-13295.

(76) Dubbeldam, D.; Beerdsen, E.; Vlugt, T. J.; Smit, B., J. Chem. Phys. 2005, 122, 17.

(77) Sarkisov, L.; Harrison, A., Mol. Simul. 2o11, 37 (15), 1248-1257. 


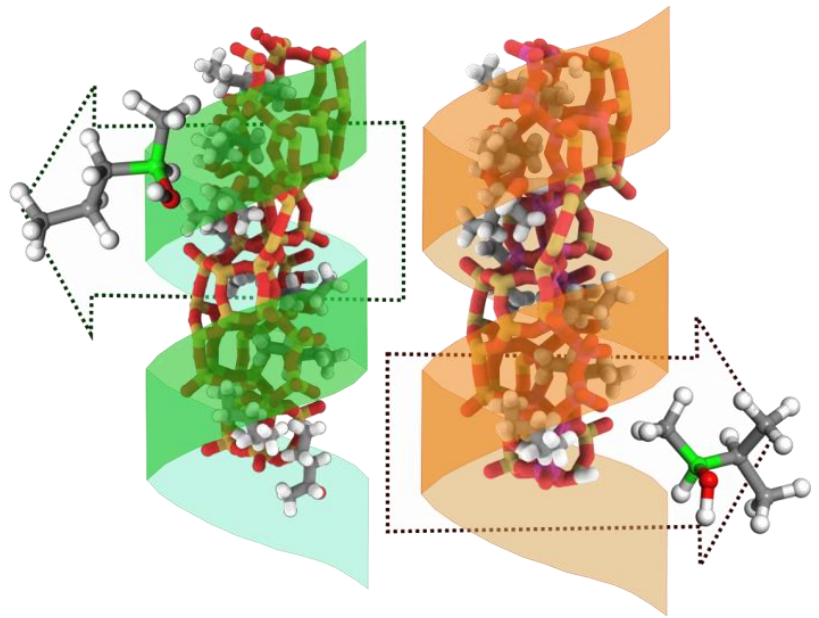

